\title{
DIRECT TWO-PHOTON CREATION OF EXCITONIC MOLECULE IN $\mathrm{CuCl}$
}

\author{
W. Ungier, P. Janiszewski and M. SufFCZYŃsKi \\ Institute of Physics, Polish Academy of Sciences \\ Al. Lotników 32/46, 02-668 Warszawa, Poland \\ (Received May 9, 2000; revised version July 21, 2000)
}

\begin{abstract}
The probability of direct excitation of excitonic molecule in the two-photon absorption process is calculated with an Hylleraas-Ore type biexciton envelope, which is variationally optimized. In the second order of perturbation, because of the resonance effect, only the exciton $\Gamma_{5}$ intermediate state is taken into account. The band structure at band extrema due to spin-orbit interaction is assumed. The transition probability of two-photon absorption is expressed by matrix element between the free exciton and the biexciton envelopes. The obtained probability of two-photon absorption in $\mathrm{CuCl}$ is about three orders of magnitude smaller when compared to that obtained by Hanamura.
\end{abstract}

PACS numbers: 71.35.-y

\section{Introduction}

A shallow exciton can be regarded as a bound state of an electron in the conduction band and a positive hole in the valence band. Adamowski et al. [1] and independently Akimoto and Hanamura [2] pointed out that two weakly bound excitons form a biexciton (similarly to two hydrogen atoms which form a hydrogen molecule) for any value of the electron-hole mass ratio. The existence of the biexcitons has been confirmed in CdS, CdSe [3], in $\mathrm{CuCl}, \mathrm{CuBr}$ [4] by the emission spectrum under strong excitation. The Bose condensation of these excitonic molecules [5] was also observed on the emission spectrum under the picosecond laser pulse excitation. Hanamura pointed out that the two-photon absorption due to excitonic molecule is enhanced due to the same effect of giant oscillator strength as in bound exciton [6] and the resonance effect.

We now present a more detailed estimation of the two-photon absorption coefficient due to excitonic molecule. In $\mathrm{CuCl}$, compared with $\mathrm{CuBr}$, valence band levels $\Gamma_{7}$ and $\Gamma_{8}$ are inverted. In $\mathrm{CuCl}$ the uppermost valence band maximum has symmetry $\Gamma_{7}$. It is much more simply to consider biexciton formed with the doubly degenerate hole states $\Gamma_{7}$ than that formed with the fourfold degenerate states $\Gamma_{8}$.

In our calculations we use the biexciton envelope optimized by variational calculation. 


\section{Two-photon excitation probability}

The transition probability for the two $\omega$-photon excitation of an insulating crystal from its ground state $|g\rangle$ to the excited state $\mid$ biex $\rangle$ with an excitonic molecule [7] is

$$
W^{(2)}(\omega)=\frac{2 \pi}{\hbar} A^{2}\left\langle\text { biex }\left|\varepsilon \sum_{j} \boldsymbol{P}_{j} \sum_{i} \frac{|i\rangle\langle i|}{E_{i g}-\hbar \omega} \varepsilon \sum_{j^{\prime}} \boldsymbol{P}_{j^{\prime}}\right| g\right\rangle \delta\left(2 \hbar \omega-E_{\mathrm{mol}}\right),
$$

where $A=(e / m)^{2}(2 \pi \hbar N / \kappa \omega V)$ and $N$ is the number of $\omega$ photons in the incident beam with polarization $\varepsilon, \kappa$ is a dielectric constant for frequency $\omega$. $V$ is the volume of the crystal and $\boldsymbol{P}_{j}=\exp \left(\mathrm{i} \boldsymbol{K}_{0} \boldsymbol{r}_{j}\right) \boldsymbol{p}_{j}$ with $\boldsymbol{p}_{j}$ - the momentum of the $j$-th electron and $\boldsymbol{K}_{0}$ - the wave number vector of light. We count the energy from the ground state $|g\rangle$. We consider the ground state of the biexciton with energy $E_{\text {mol }}\left(\Gamma_{1}\right)$. The intermediate states are the excitonic states $|i\rangle$. By paying attention to the resonance effect we will only need to take the lowest intermediate excitonic states with energy $E_{\text {ex }}\left(\Gamma_{5}\right)$.

\section{Excitations in $\mathrm{CuCl}$ with zinc blende structure}

Cuprous chloride, $\mathrm{CuCl}$ - the crystal with tetrahedral symmetry (group $T_{d}$ ) has a band structure with the top of the valence band at $k=0$ of symmetry $\Gamma_{7}$ and the bottom of the conduction band at $k=0$ of symmetry $\Gamma_{6}$ (we use notation of Koster et al. [8]). The conduction Bloch functions $\left(\Gamma_{6}\right)$ can be written as

$$
c_{1 / 2}=\phi_{c}|\uparrow\rangle \text { and } c_{-1 / 2}=\phi_{c}|\downarrow\rangle
$$

where $\phi_{c}$ can be identified with the $4 s$-functions of $\mathrm{Cu}$. The valence Bloch functions $\left(\Gamma_{7}\right)$ are of the form

$$
v_{1 / 2}=\psi_{1}|\downarrow\rangle+\psi_{0}|\uparrow\rangle \text { and } v_{-1 / 2}=\psi_{-1}|\uparrow\rangle-\psi_{0}|\downarrow\rangle,
$$

where the top valence wave functions may be identified with $3 p$-functions of $\mathrm{Cl}$ :

$$
\psi_{ \pm 1}=(1 / \sqrt{3})\left(p_{x} \pm \mathrm{i} p_{y}\right), \quad \psi_{0}=(1 / \sqrt{3}) p_{z} .
$$

Here $p_{x}, p_{y}, p_{z}$ are separately normalized. The states $|\uparrow\rangle$ and $|\downarrow\rangle$ are the pure spin eigenstates corresponding to $\hbar / 2$ and $-\hbar / 2$, respectively.

In the effective mass approximation we replace the periodic parts of the Bloch functions by their values at the band extrema $c_{ \pm 1 / 2}$ and $v_{ \pm 1 / 2}$.

In $\mathrm{CuCl}$ the crystal symmetries of the biexciton states are given by the following product of irreducible representations:

$$
\Gamma_{6} \otimes \Gamma_{7}^{*} \otimes \Gamma_{6} \otimes \Gamma_{7}^{*}=\left(\Gamma_{2}+\Gamma_{5}\right) \otimes\left(\Gamma_{2}+\Gamma_{5}\right)=2 \Gamma_{1}+3 \Gamma_{4}+\Gamma_{3}+\Gamma_{5} .
$$

In the total momentum classification of the exciton states, $\Gamma_{2}$ corresponds to $J=0, \Gamma_{5}$ to $J=1$. The energy of the exciton $\Gamma_{5}$ is increased by the electron-hole exchange interaction. In the classification of the biexciton states $\Gamma_{1}$ corresponds to $J=0, \Gamma_{4}$ to $J=1$ and $\Gamma_{3}+\Gamma_{5}$ results from the splitting of $J=2$.

We will consider the optical two-photon transition from the ground state $|g\rangle$ to the biexciton ground state of symmetry $\Gamma_{1}$. Because the optical excitations from the state $|g\rangle$ to the exciton $\Gamma_{1}(J=0)$ are forbidden, we will take as the 
intermediate states the nearest exciton states of symmetry $\Gamma_{5}(J=1)$. The excited two-particle (one electron and one hole) triplet states [9] can be expressed with the use of the fermion operators

$$
\begin{aligned}
\mid l, k ; J & \left.=1, J_{z}\right\rangle \\
& = \begin{cases}a_{c l, 1 / 2}^{+} a_{v k,-1 / 2}|g\rangle & \left(J_{z}=1\right), \\
(1 / \sqrt{2})\left(a_{c l, 1 / 2}^{+} a_{v k, 1 / 2}-a_{c l,-1 / 2}^{+} a_{v k,-1 / 2}\right)|g\rangle & \left(J_{z}=0\right), \\
a_{c l,-1 / 2}^{+} a_{v k, 1 / 2}|g\rangle & \left(J_{z}=-1\right) .\end{cases}
\end{aligned}
$$

The operator $a_{c l, \pm 1 / 2}^{+}$creates the electron in the conduction band in the Bloch state with the wave vector $l$ and $J_{z}= \pm 1 / 2$, while $a_{v k, \pm 1 / 2}$ annihilates the electron in the valence band in the Bloch state with the wave vector $k$ and $J_{z}= \pm 1 / 2$.

We assume the exciton $\Gamma_{5}$ states in the form of a linear combination

$$
\left|\Gamma_{5}^{\mathrm{ex}} ; \boldsymbol{K}, J_{z}\right\rangle=\sum_{\boldsymbol{l}, \boldsymbol{k}^{\prime}} A_{\boldsymbol{K}}(\boldsymbol{l}, k)\left|\boldsymbol{l}, k ; J=1, J_{z}\right\rangle .
$$

The summation over the wave vectors $l, k$ is limited here to $l-k=K$, the total wave vector of the free exciton center of mass. The linear coefficients $A_{\boldsymbol{K}}(l, k)$ are the Fourier components of the envelope

$$
A_{\boldsymbol{K}}(\boldsymbol{l}, \boldsymbol{k})=\frac{1}{V} \iint \mathrm{d}^{3} r_{1} \mathrm{~d}^{3} r_{2} \phi_{\boldsymbol{K}}\left(\boldsymbol{r}_{2}, \boldsymbol{r}_{2}\right) \exp \left(-\mathrm{i} \boldsymbol{l} \boldsymbol{r}_{1}+\mathrm{i} \boldsymbol{k} r_{2}\right),
$$

where $\phi_{\boldsymbol{K}}$ is the free-exciton envelope function [10]

$$
\phi_{\boldsymbol{K}}\left(\boldsymbol{r}_{1}, \boldsymbol{r}_{2}\right)=\frac{1}{\sqrt{V}} \exp \left(\mathrm{i} \boldsymbol{K} \boldsymbol{R}_{0}^{(\mathrm{ex})}\right) \Phi_{\mathrm{ex}}(\boldsymbol{r}) .
$$

The exciton envelope $\Phi_{\mathrm{ex}}(r)$ is a function of the electron-hole distance $r=\left|\boldsymbol{r}_{1}-\boldsymbol{r}_{2}\right|$, $\boldsymbol{R}_{0}^{(\mathrm{ex})}=\left(m_{\mathrm{e}} \boldsymbol{r}_{1}+m_{\mathrm{h}} \boldsymbol{r}_{2}\right) / M$ is the position of exciton centre of mass, $m_{\mathrm{e}}, m_{\mathrm{h}}$ are the electron and hole effective masses and $M=m_{\mathrm{e}}+m_{\mathrm{h}} . V$ is the volume of the crystal.

The biexciton ground state of the symmetry $\Gamma_{1}$ is described by a spatial envelope function symmetrized with respect to the permutation of two electrons, as well as the permutation of two holes [11, 12]. According to this symmetry we define the excited four particle singlet states symmetric in $l, l^{\prime}$ and symmetric in $k, k^{\prime}$ :

$$
\begin{aligned}
& \left|l, k, l^{\prime}, k^{\prime} ; J=0\right\rangle=\frac{1}{2}\left(a_{c l, 1 / 2}^{+} a_{c l^{\prime},-1 / 2}^{+}-a_{c l,-1 / 2}^{+} a_{c l^{\prime}, 1 / 2}^{+}\right) \\
& \times\left(a_{v k, 1 / 2} a_{v k^{\prime},-1 / 2}-a_{v k,-1 / 2} a_{v k^{\prime}, 1 / 2}\right)|g\rangle .
\end{aligned}
$$

We assume the biexciton ground state in the form

$$
\left|\Gamma_{1}^{\mathrm{mol}} ; \boldsymbol{K}\right\rangle=\sum_{\boldsymbol{l}, \boldsymbol{k}, \boldsymbol{l}^{\prime}, \boldsymbol{k}^{\prime}} B_{\boldsymbol{K}}\left(\boldsymbol{l}, \boldsymbol{k}, \boldsymbol{l}^{\prime}, k^{\prime}\right)\left|l, k, \boldsymbol{l}^{\prime}, k^{\prime} ; J=0\right\rangle .
$$

Here the summation is limited to $l-k+l^{\prime}-k^{\prime}=K$, where $K$ is the total momentum of the biexciton. The linear coefficients $B_{\boldsymbol{K}}$ are the Fourier components 


$$
\begin{aligned}
& B_{\boldsymbol{K}}\left(l, k, l^{\prime}, k^{\prime}\right)=\frac{1}{V^{2}} \iiint \int \mathrm{d}^{3} r_{1} \mathrm{~d}^{3} r_{2} \mathrm{~d}^{3} r_{3} \mathrm{~d}^{3} r_{4} \psi_{\boldsymbol{K}}\left(\boldsymbol{r}_{1}, \boldsymbol{r}_{2}, r_{3}, r_{4}\right) \\
& \quad \times \exp \left(-\mathrm{i} l \boldsymbol{r}_{1}+\mathrm{i} k r_{2}-\mathrm{i} l^{\prime} r_{3}+\mathrm{i} k^{\prime} r_{4}\right),
\end{aligned}
$$

where $\psi_{\boldsymbol{K}}$ is the biexciton wave function of the electron $(1,3)$ and the hole $(2,4)$ coordinates

$$
\psi_{\boldsymbol{K}}\left(\boldsymbol{r}_{1}, \boldsymbol{r}_{2}, \boldsymbol{r}_{3}, \boldsymbol{r}_{4}\right)=\frac{1}{2 \sqrt{V}} \exp \left(\mathrm{i} \boldsymbol{K} \boldsymbol{R}_{0}^{\mathrm{mol}}\right) \Psi_{\mathrm{mol}}\left(\boldsymbol{r}, \boldsymbol{r}^{\prime}, \boldsymbol{R}\right) .
$$

Here $\boldsymbol{R}_{0}^{\mathrm{mol}}=m_{\mathrm{e}}\left(\boldsymbol{r}_{1}+r_{3}\right) / 2 M+m_{\mathrm{h}}\left(\boldsymbol{r}_{2}+r_{4}\right) / 2 M$ is the position of biexciton centre of mass and $\Psi$ is the biexciton ground state envelope function [10] of $r=r_{3}-r_{1}, r^{\prime}=$ $r_{4}-r_{2}$ and $\boldsymbol{R}=\left(r_{1}+r_{3}-r_{2}-r_{4}\right) / 2$. Introducing the factor $1 /(2 \sqrt{V})$ in Eq. (13) we have for the normalized biexciton state (10) the normalization condition of the envelope function $\Psi: \int \mathrm{d}^{3} r \int \mathrm{d}^{3} r^{\prime} \int \mathrm{d}^{3} R\left|\Psi_{\text {mol }}\left(r, r^{\prime}, R\right)\right|^{2}=1$.

\section{The two-photon absorption}

For the transition with absorption of light we use in the zinc blende type $\mathrm{CuCl}$ crystal the customary dipole approximation

$$
\begin{aligned}
\varepsilon \sum_{j=1}^{N} & \exp \left(\mathrm{i} \boldsymbol{K}_{0} \boldsymbol{r}_{j}\right) \boldsymbol{p}_{j}=\varepsilon \sum_{\lambda}\left(\left\langle\phi_{c}|p| \psi_{-1}\right\rangle a_{c \lambda+K_{0}, 1 / 2}^{+} a_{v \lambda,-1 / 2}\right. \\
& +\left\langle\phi_{c}|\boldsymbol{p}| \psi_{0}\right\rangle a_{c \lambda+K_{0}, 1 / 2}^{+} a_{v \lambda, 1 / 2}+\left\langle\phi_{c}|p| \psi_{1}\right\rangle a_{c \lambda+K_{0},-1 / 2}^{+} a_{v \lambda, 1 / 2} \\
& \left.\quad\left\langle\phi_{c}|\boldsymbol{p}| \psi_{0}\right\rangle a_{c \lambda+K_{0},-1 / 2}^{+} a_{v \lambda,-1 / 2}\right),
\end{aligned}
$$

where $p_{j}$ is the momentum of $j$-th electron and $\left\langle\phi_{c}|p| \psi_{J_{z}}\right\rangle$ is the matrix element of the electron momentum $p$ between the valence and the conduction band wave functions.

The matrix elements in Eq. (1) are evaluated, with the use of the approximation (14), as follows:

$$
\begin{aligned}
& \left\langle\Gamma_{5}^{\mathrm{ex}} ; \boldsymbol{K}, J_{z}\left|\varepsilon \sum_{j=1}^{N} \exp \left(\mathrm{i} \boldsymbol{K}_{0} \boldsymbol{r}_{j}\right) \boldsymbol{p}_{j}\right| g\right\rangle \\
& =\left\{\begin{array}{l}
\delta_{\boldsymbol{K}_{,} \boldsymbol{K}_{0}} \sqrt{V} \Phi_{\mathrm{ex}}(0)\left\langle\phi_{c}|\varepsilon p| \psi_{\mp 1}\right\rangle \text { for } J_{z}= \pm 1 \\
\delta_{\boldsymbol{K}, \boldsymbol{K}_{0}} \sqrt{V} \Phi_{\mathrm{ex}}(0) \sqrt{2}\left\langle\phi_{c}|\varepsilon p| \psi_{0}\right\rangle \text { for } J_{z}=0
\end{array}\right.
\end{aligned}
$$

and

$$
\begin{aligned}
& \left\langle\Gamma_{1}^{\mathrm{mol}} ; \boldsymbol{K}\left|\varepsilon \sum_{j=1}^{N} \exp \left(\mathrm{i} \boldsymbol{K}_{0} r_{j}\right) p_{j}\right| \Gamma_{5}^{\mathrm{exp}} ; \boldsymbol{K}_{0}, J_{z}\right\rangle \\
& \quad=\left\{\begin{array}{l}
\delta_{\boldsymbol{K}, 2 \boldsymbol{K}_{0}} I\left[\Phi_{\mathrm{ex}}, \Psi_{\mathrm{mol}}\right]\left\langle\phi_{c}|\boldsymbol{\varepsilon} p| \psi_{ \pm}\right\rangle \text {for } J_{z}= \pm 1, \\
\delta_{\boldsymbol{K}, 2 \boldsymbol{K}_{0}} I\left[\Phi_{\mathrm{ex}}, \Psi_{\mathrm{mol}}\right] \sqrt{2}\left\langle\phi_{c}|\boldsymbol{\varepsilon}| \psi_{0}\right\rangle \text { for } J_{z}=0,
\end{array}\right.
\end{aligned}
$$

where for $\boldsymbol{K}_{0} \approx 0$ the functional of the exciton and biexciton envelopes is defined 


$$
\begin{aligned}
& I\left[\Phi_{\mathrm{ex}}, \Psi_{\mathrm{mol}}\right]=2 \sum_{\boldsymbol{l}, \boldsymbol{k}} B_{2 K_{0}}^{*}\left(l, l-K_{0}, k+K_{0}, k\right) A_{K_{0}}\left(l, l-K_{0}\right) \\
& \quad \approx \iint \mathrm{d}^{3} x \mathrm{~d}^{3} y \Psi_{\mathrm{mol}}^{*}(x-y, x, y / 2) \Phi_{\mathrm{ex}}(y) .
\end{aligned}
$$

Inserting matrix elements (15)-(16) into Eq. (1) we get for $\boldsymbol{K}_{0} \approx 0$

$$
\begin{aligned}
& W^{(2)}\left(\Gamma_{1}^{\mathrm{mol}} ; \omega\right)=\frac{2 \pi}{\hbar} A^{2}\left\{\Phi_{\mathrm{ex}}(0) \sqrt{V} I\left[\Phi_{\mathrm{ex}}, \Psi_{\mathrm{mol}}\right] \frac{1}{E_{\mathrm{ex}}\left(\Gamma_{5}\right)-\hbar \omega}\right. \\
& \left.\quad \times\left[\left\langle\phi_{c}|\varepsilon p| \psi_{1}\right\rangle\left\langle\phi_{c}|\varepsilon p| \psi_{-1}\right\rangle+\left\langle\phi_{c}|\varepsilon p| \psi_{0}\right\rangle^{2}\right]\right\}^{2} \delta\left[2 \hbar \omega-E_{\mathrm{mol}}\left(\Gamma_{1}\right)\right] .
\end{aligned}
$$

Because of the cubic symmetry $\left\langle\phi_{c}\left|p_{x}\right| p_{x}\right\rangle=\left\langle\phi_{c}\left|p_{y}\right| p_{y}\right\rangle=\left\langle\phi_{c}\left|p_{z}\right| p_{z}\right\rangle$, Eq. (18) is simplified to

$$
\begin{array}{r}
W^{(2)}\left(\Gamma_{1}^{\mathrm{mol}} ; \omega\right)=\frac{2 \pi}{\hbar} A^{2} \Phi_{\mathrm{ex}}^{2}(0) V I^{2}\left[\Phi_{\mathrm{ex}}, \Psi_{\mathrm{mol}}\right] \\
\times \frac{4 \varepsilon^{4}\left|\left\langle\phi_{c}\left|p_{x}\right| p_{x}\right\rangle\right|^{4}}{9\left[E_{\mathrm{ex}}\left(\Gamma_{5}\right)-\hbar \omega\right]^{2}} \delta\left[2 \hbar \omega-E_{\mathrm{mol}}\left(\Gamma_{1}\right)\right] .
\end{array}
$$

We will compare $W^{(2)}$ with the transition probability of one-photon absorption due to a single exciton

$$
W^{(1)}\left(\Gamma_{5}^{\mathrm{ex}} ; \omega\right)=\frac{2 \pi}{\hbar} A V \Phi_{\mathrm{ex}}^{2}(0) \frac{2}{3} \varepsilon^{2}\left|\left\langle\phi_{c}\left|p_{x}\right| p_{x}\right\rangle\right|^{2} \delta\left[\hbar \omega-E_{\mathrm{ex}}\left(\Gamma_{5}\right)\right]
$$

\section{Numerical results}

The probabilities $W^{(1)}\left(\Gamma_{5}^{\mathrm{ex}} ; \omega\right)$ at $\omega=\omega_{\mathrm{ex}}=E_{\mathrm{ex}}\left(\Gamma_{5}\right) / \hbar$ and $W^{(2)}\left(\Gamma_{1}^{\mathrm{mol}} ; \omega\right)$ at $\omega=\omega_{\mathrm{mol}}=\frac{1}{2} E_{\mathrm{mol}}\left(\Gamma_{1}\right) / \hbar=\left[E_{\mathrm{ex}}\left(\Gamma_{2}\right)-\frac{1}{2} E_{\mathrm{mol}}^{\mathrm{b}}\right] / \hbar$ with $E_{\mathrm{mol}}^{\mathrm{b}}$ - the binding energy of excitonic molecule, can be compared by taking the following experimental values for $\mathrm{CuCl}$ : $\left|\left\langle\phi_{c}\left|p_{x}\right| p_{x}\right\rangle\right|^{2} / m \approx 3 \mathrm{eV}[6], \hbar \omega=3.4 \mathrm{eV}$ and $E_{\text {ex }}\left(\Gamma_{5}\right)-\hbar \omega=$ $\frac{1}{2} E_{\mathrm{mol}}^{\mathrm{b}}+\Delta_{\mathrm{ex}}^{\mathrm{exch}}$ with $E_{\mathrm{mol}}^{\mathrm{b}} \approx 30 \mathrm{meV}$ and $\Delta_{\mathrm{ex}}^{\mathrm{exch}}=E_{\mathrm{ex}}\left(\Gamma_{5}\right)-E_{\mathrm{ex}}\left(\Gamma_{2}\right) \approx 6 \mathrm{meV}$ [13].

The integral $I=I\left[\Phi_{\mathrm{ex}}, \Psi_{\mathrm{mol}}\right]$ is equal to the square root of the ratio of the oscillator strengths due to the transition of the biexciton-free exciton and to the recombination of the free exciton. We take the value $I \approx 4$ obtained by one of the authors [14] with the optimized envelope $\Psi_{\text {mol }}$. Function $\Psi_{\text {mol }}$ was approximated by the Hylleraas-Ore $[15,16]$ wave function $\psi$ of electron-hole distances $r_{i j}$. All the distances are expressed in units of exciton Bohr radius $a_{0}=\kappa_{0}^{2} / \mu e^{2}$, where $\kappa_{0}$ is crystal's dielectric constant and $\mu=m_{\mathrm{e}} m_{\mathrm{h}} /\left(m_{\mathrm{e}}+m_{\mathrm{h}}\right)$ is the reduced mass of the exciton. The envelope is $\Psi_{\mathrm{mol}}=\psi F / S$ with $\psi=$ $\left.2 \exp \left[-\left(s_{1}+s_{2}\right) / 2\right)\right] \cosh \left[\beta\left(t_{1}-t_{2}\right) / 2\right]$, where $s_{1}=k\left(r_{12}+r_{14}\right), s_{2}=k\left(r_{32}+r_{34}\right)$, $t_{1}=k\left(r_{12}-r_{14}\right), t_{2}=k\left(r_{32}-r_{34}\right), F / S$ is the function of the hole-hole distance $\rho=r_{24}$ introduced by Brinkman et al. [11]: $F(\rho)=(\rho / A)^{n} \exp (-\rho / A)+$ $C \exp (-B \rho / A)$ and $S^{2}(\rho)=\int \psi^{2} \mathrm{~d}^{3} r_{1} \mathrm{~d}^{3} r_{2}$. The variational parameters of $\Psi_{\mathrm{mol}}(k, \beta, A, B, C, n)$ depend on the ratio of effective masses $\sigma=m_{\mathrm{e}} / m_{\mathrm{h}}$ (for $\mathrm{CuCl} \sigma=0.02$ ). 
The exciton envelope $\Phi_{\mathrm{ex}}$ was taken in the form of the hydrogen-like wave function $\Phi_{\mathrm{ex}}(r)=\exp (-r) / \sqrt{\pi}$. We have obtained the ratio of the probabilities $W^{(2)} / W^{(1)}=3.5 \times 10^{-18} \mathrm{~cm}^{3} \mathrm{~N} / \mathrm{V}$. Our result is smaller than that previously obtained by Hanamura $W^{(2)} / W^{(1)}=3 \times 10^{-15} \mathrm{~cm}^{3} \mathrm{~N} / \mathrm{V}$. In comparison with an ordinary two-photon absorption due to a single electron excitation there are two enhancement factors determining direct two-photon absorption probability $W^{(2)}(19): I^{2}\left[\Phi_{\mathrm{ex}}, \Psi_{\mathrm{mol}}\right]$, which corresponds to the oscillator strength [14], and the second one, which comes from the resonance effect $1 /\left[E_{\mathrm{ex}}\left(\Gamma_{5}\right)-\hbar \omega\right]^{2} . I\left[\Phi_{\mathrm{ex}}, \Psi_{\mathrm{mol}}\right]$ obtained with the use of the optimized envelope [14] is about 10 times smaller compared to that estimated by Ueta [6]. The resonance denominator, corrected by the electron-hole exchange term is twice greater than that used in [6].

Therefore at the dye laser of tunable frequency above the photon density $N / V=10^{17} \mathrm{~cm}^{-3}$, the two-photon absorption coefficient at $\omega=\omega_{\mathrm{mol}}$ is expected to be as strong as a single excitation absorption at $\omega=\omega_{\text {ex }}$.

We note that time of biexciton decay corresponding to the oscillator strength of biexciton into exciton transition estimated with simple envelope used by Hanamura was too small $[16,17]$. Therefore the smaller value of the integral $I\left[\Phi_{\mathrm{ex}}, \Phi_{\mathrm{mol}}\right]$ and thus, the smaller than previously estimated value of $W^{(2)} / W^{(1)}$ seems to be more appropriate.

We also estimated the ratio $W^{(2)} / W^{(1)}$ in case of $\mathrm{CuBr}$ crystal. Taking the electron to hole mass ratio $\sigma=0.01[11]$ we got $W^{(2)} / W^{(1)} \approx 10^{-18} \div 10^{-17}$ $\mathrm{cm}^{3} \mathrm{~N} / \mathrm{V}$, which is close to the result obtained for $\mathrm{CuCl}$.

\section{References}

[1] J. Adamowski, S. Bednarek, M. Suffczyński, Solid State Commun. 9, 2037 (1971).

[2] O. Akimoto, E. Hanamura, Solid State Commun. 10, 253 (1972).

[3] S. Shionoya, H. Saito, E. Hanamura, O. Akimoto, Solid State Commun. 12, 223 (1973).

[4] A. Mysyrowicz, J.B. Grun, R. Levy, A. Bivas, S. Nikitine, Phys. Lett. A 26, 615 (1968).

[5] H. Kuroda, S. Shionoya, H. Saito, E. Hanamura, Solid State Commun. 12, 533 (1973).

[6] M. Ueta, H. Kanzaki, K. Kobayashi, Y. Toyozawa, E. Hanamura, in: Excitonic Processes in Solids, Springer, Berlin 1986, p. 63.

[7] M. Inoue, Y. Toyozawa, J. Phys. Soc. Jpn. 20, 363 (1965).

[8] K.F. Koster, J.O. Dimmock, R.G. Wheeler, H. Statz, Properties of the Thirty Two Point Groups, M.I.T. Press, Cambridge, Massachusetts 1963.

[9] F. Bassani, G. Pastori Parravicini, Electronic States and Optical Transitions in Solids, Pergamon Press, Oxford 1975, p. 185.

[10] J.O. Dimmock, Semiconductors and Semimetals, Eds. R.K. Willardson, A.C. Beer, Vol. 3, Academic Press, New York 1967, p. 259.

[11] W.F. Brinkman, T.M. Rice, B. Bell, Phys. Rev. B 8, 1570 (1973).

[12] F. Bassani, J.J. Forney, A. Quattropani, Phys. Status Solidi B 65, 591 (1974).

[13] M. Certier, C. Wecker, S. Nikitine, J. Phys. Chem. Solids 30, 2135 (1969). 
[14] W. Ungier, Solid State Commun. 110, 639 (1999).

[15] E.A. Hylleraas, A. Ore, Phys. Rev. 71, 493 (1947).

[16] W. Ungier, M. Suffczyński, J. Quant. Chem. 60, 1001 (1996).

[17] D. Birdekal, J. Singh, V.G. Lyssenko, J. Erland, J.M. Hvam, Phys. Rev. Lett. 76, $672(1996)$. 